\title{
P03-14-1 Poster session
}

\section{The effect of CYP2C19*17 on platelet aggregation in patients with acute coronary syndrome and percutaneous coronary intervention in Russian population}

\author{
Kristina Ryzhikova ${ }^{1}$, Karin Mirzaev ${ }^{1}$ Elena Grishina ${ }^{1}$, Denis Andreev², Dmitrij Sychev ${ }^{1}$ \\ ${ }^{I}$ Russian Medical Academy of Continuous Professional Education, Moscow, Russia, ${ }^{2}$ First Moscow State Medical \\ University, Russia
}

Background

The aim of this study was to assess the impact of CYP2C19*17 on low platelet reactivity (PRU $<85$ for VerifyNow assay) in clopidogrel-treated patients with acute coronary syndrome (ACS) and percutaneous coronary intervention (PCI).

Material and methods

A total of 81 (64 males and 17 females) patients with ACS and PCI participated in the study. The mean age of patients enrolled was $63.9 \pm 10.9$ years. We measured platelet reactivity using VerifyNow P2Y12 assay. Genomic DNA was extracted from venous blood. CYP2C19*17 alleles was determined by real-time polymerase chain reaction (Real-Time PCR). Fisher's exact test was used to assess CYP2C19*17 allele frequency between patient with normal platelets reactivity $(\mathrm{PRU}>85)$ and low platelets reactivity $(\mathrm{PRU}<85)$. A P-value $<0.05$ was considered statistically significant.

Results

From the 81 patients, included in research and genotyping by CYP2C19*17,57(70\%) were wild type homozygous (CC), $23(28 \%)$ were heterozygotes (CT) and $1(2 \%)$ was homozygous for the T-allelic variant (TT). C-allele frequency was 84.6\% and incidence of T-allele was $15.4 \%$. Genotype frequencies didn ' $t$ deviate significantly from Hardy-Weinberg equilibrium ( $\mathrm{p}=0.42, \mathrm{x} 2=0.06$ ). There were statistically significant differences in CYP2C19*17 allele frequency between patients with low platelet reactivity $(\mathrm{LPR})(\mathrm{PRU}<85)$ and in patients with a normal platelet reactivity $(\mathrm{PRU}>85)$ : $\mathrm{OR}$, 11.2; $95 \%$ CI, 1.18 to $106.32 ; \mathrm{p}=0.0253)$.

Conclusion

Carriers of CYP2C19*17 allelic variant have the higher risk of developing LPR and risk of bleeding events, respectively. Futher research with large data is needed to prove the result of this study. 